MS29-03

\section{Bilbao Crystallographic Server - new databases and computer tools for the study of layer and multilayer materials}

Gemma de la Flor Martin', Danel Orobengoa' ${ }^{1}$, Yuri E. Kitaev², Robert Evarestov ${ }^{3}$, Luis Elcoro ${ }^{1}$, Gotzon Madariaga ${ }^{1}$, Mois I. Aroyo ${ }^{1}$

1. Departamento Física de la Materia Condensada, Universidad del País Vasco UPV/EHU, Bilbao, Spain

2. Laboratory of Quantum-size Hererostructures, Ioffe Institute, Saint Petersburg, Russia

3. Institute of Chemistry, Saint Petersburg State University, Saint Petersburg, Russia

email: gemma.delaflor@ehu.es

The Bilbao Crystallographic Server (www.cryst.ehu.es) [1$2]$ is a free web site that grants access to specialized databases and tools for the resolution of different types of crystallographic, structural chemistry and solid-state physics problems. The server is in constant improvement and development, offering free of charge tools to study an increasingly number of crystallographic systems. Recently, new tools dedicated to the study of materials with layer and multilayer symmetry have been developed due to the arising interest in these type of materials. The purpose of this contribution is to report on the current state of the server emphasizing on the recently developed computer tools for the study of materials with layer group symmetry. The utility of the available applications will be demonstrated by illustrative examples.

The section Subperiodic groups: Layer, Rod and Frieze Groups gives access to the layer groups databases containing the basic crystallographic information, and the Brillouin-zone and $\mathbf{k}$-vectors tables that form the background and a classification of the irreducible representations (irreps) of the 80 layer groups. The programs GENPOS, WPOS, MAXSUB and KVEC provide access to the general positions, Wyckoff positions, maximal subgroups and Brillouin-zone databases, respectively. More complex tools such as the program SECTIONS that identifies the layer symmetry of space-group sections are also accessible in this section. This program calculates the full set of possible layer symmetries of periodic sections defined by their common normal vector and located within the unit cell for a given space group. The last tool to join this section is the program LSITESYM which is based on the so-called site-symmetry approach [3] that established symmetry relations between the localized state (local atomic displacements) and crystal extended states (phonon, electrons, etc.) over the entire Brillouin zone. The method is based on the procedure of induction of representations of the layer group of the crystal from the irreps of the site-symmetry groups of constituent units (atoms, clusters and layers) according to which the local excitations are transformed. For a given layer group, $\mathbf{k}$-vector and a selected representative of a Wyckoff position, the program LSITESYM returns the symmetry operations of the site symmetry group, its irreps and the irreps of the crystal layer group according to which transform the compatible extended states.
References:

[1] M. I. Aroyo et al. (2006) Z. Kristallog., 221, 15-27

[2] M. I. Aroyo et al. (2011) Bulgarian Chemical Communications, 43(2), 183-197

[3] A . Evarestov and V. P. Smirnov, Site Symmetry in Crystals. Theory and Applications, Springer Series in Solid State Science, ed. By M. Cardina (Springer, Berlin, 993), 2nd ed. 1997

Keywords: subperiodic groups, bilbao crystallographic server, symmetry 